Review

\title{
Evaluation of condylar resorption rates after orthognathic surgery in class II and III dentofacial deformities: A systematic review
}

\author{
Valthierre Nunes de Lima ${ }^{\text {a, }}$, Leonardo Perez Faverani ${ }^{a}$, Joel Ferreira Santiago Jr. ${ }^{b}$, \\ Celso Palmieri Jr. ${ }^{\text {, }}$ Osvaldo Magro Filho ${ }^{a}$, Eduardo Piza Pellizzer ${ }^{\text {a }}$ \\ a São Paulo State University (Unesp), School of Dentistry, Araçatuba, São Paulo, Brazil \\ b Sacred Heart University, School of Dentistry, Bauru, São Paulo, Brazil \\ ${ }^{\mathrm{c}}$ Department of Oral \& Maxillofacial Surgery, Louisiana State University Health Sciences Center, Shreveport, LA, USA
}

\section{A R T I C L E I N F O}

\section{Article history:}

Paper received 5 October 2017

Accepted 7 February 2018

Available online 21 February 2018

\section{Keywords:}

Orthognathic surgery

Mandibular condyle

Temporomandibular joint

\begin{abstract}
A B S T R A C T
The purpose of this study was to perform a systematic review of morphological alterations in the condyles after orthographic surgery involving a sagittal split ramus osteotomy (SSRO), with or without surgery on the maxilla. Searches were performed on three databases and registered in the PROSPERO. The selected studies fulfilled the criteria established by the following PICO model: (1) population: individuals with skeletal dentofacial deformities (class II or III facial patterns), without asymmetry; (2) intervention: orthognathic surgery for mandibular setback using an SSRO, with or without a Le Fort I osteotomy, and fixed with bicortical screws or plates and screws; (3) comparison: orthognathic surgery for mandibular advancement using an SSRO, with or without a Le Fort I osteotomy, and fixed with plates and screws or bicortical screws; and (4) outcome: condylar resorption rate and relapse. Initially, 1,371 articles were identified and 636 articles were screened after elimination of duplicates, and 6 articles were selected for qualitative analysis based on the inclusion and exclusion criteria. Five studies had data regarding the rate of condylar resorption, varying from $0.0 \%$ to $4.2 \%$. In conclusion, condylar resorption and relapses were present in a small percentage of patients studied.
\end{abstract}

(C) 2018 European Association for Cranio-Maxillo-Facial Surgery. Published by Elsevier Ltd. All rights reserved.

\section{Introduction}

Orthognathic surgery is performed by oral and maxillofacial surgeons in order to correct dentofacial deformities that alter the function and aesthetics of patients. There are distinct ways to perform the surgeries, however all involve osteotomies and fixations (Obwegeser, 2007).

The type and shape of fixation used for repositioning the condyles are significant. If the fixation generates torque in the condyles, the temporomandibular joint (TMJ) may undergo changes leading to condylar resorption, pain, malocclusion, and dysfunctional TMJ disorders (TMDs) (Yoshioka et al., 2008).

According to the magnitude and rate of progression, the postoperative morphological alterations of the condyles may be physiological or pathological (Arnett et al., 1996a, 1996b). In contrast to

\footnotetext{
* Corresponding author. Rua Jose Bonifacio, 1193. Cep: 16015-050, Aracatuba, São Paulo, Brazil. Fax: +55 453636 3270, +55 18981051320

E-mail address: valthierre@hotmail.com (V. Nunes de Lima).
}

the self-limiting and physiological form of condylar remodeling, patients with progressive pathological condylar resorption can experience postoperative relapse, an anterior open bite, decreased facial height, and TMDs (Hoppenreijs et al., 1998; Park et al., 2012). The distinction between condylar remodeling and condylar resorption is sometimes difficult to determine and requires evaluation using imaging (Tsiklakis et al., 2004; Billiau et al., 2007; Hussain et al., 2008; Katakami et al., 2008).

The purpose of this study was to perform a systematic review of the morphological alteration rates of the condyles after orthographic surgery involving the mandible, in patients who underwent a sagittal split ramus osteotomy (SSRO), with or without associated procedures on the maxilla, and the clinical correlations.

\section{Methods}

The systematic review was performed according to the PRISMA guidelines (Moher et al., 2009) and following the previously proposed models (Pires et al., 2016; Silva et al., 2016). It was registered 
in the PROSPERO with number CRD42016048665. Selection of the articles was completed by two authors (VNL and LPF) and a third reviewer (O.M.F.).

\subsection{Selection criteria}

The studies selected for this review met the criteria established by the following PICO model: (1) population: individuals with skeletal dentofacial deformities (class II or class III), without asymmetry; (2) intervention: orthognathic surgery for mandibular setback using an SSRO, with or without an associated Le Fort I osteotomy, and fixed with bicortical screws or plates and monocortical screws; (3) comparison: orthognathic surgery for mandibular advancement using an SSRO, with or without a Le Fort I osteotomy, and fixed with bicortical screws or plates and monocortical screws; and (4) outcome: condylar resorption rate.

\subsection{Databases}

The articles were selected from the PubMed/MEDLINE, Cochrane Library, and Embase.

\subsection{Searches}

The searches were conducted using two strategies with the following descriptors: 'orthognathic surgery' AND 'temporomandibular joint' and 'orthognathic surgery' AND 'mandibular condyle.'

\subsection{Selection of articles}

The inclusion criteria were as follows: articles written in the English language; controlled and randomized clinical trials (RCTs), prospective studies, and retrospective studies; and articles published in the last 10 years.

The exclusion criteria were as follows: animal studies, case reports, literature and systematic reviews, case series, and metaanalyses; studies on patients with the presence of asymmetries; and articles that did not present relevant data for the purpose of this study.

\subsection{Data collection process}

The articles were selected by the authors (VNL and LPF). We performed inter-examiner (Kappa) tests on each article to evaluate the title and abstract, in addition to a full reading of the article for interpretation. Kappa test agreement was present when $\mathrm{K}=0.90$; $1 ; 1$. An agreement was reached during a meeting where differences between the two authors were discussed and resolved by the third reviewer (C.A.A.L). After analysis of the titles and abstracts based on the inclusion criteria, six articles were selected.

\subsection{Data}

The following data were identified for each article: first author, year of publication, type of study, level of evidence, study time, number of patients, skeletal deformity pattern, gender, mean age, type of osteotomy, type of osteotomy fixation, postoperative maxillo-mandibular fixation (MMF) time, follow-up, imaging examination, software used for analysis, form of analysis, condylar resorption rate and relapse.

For analysis of the resorption rate in studies that included measurements of width and height of the condyles, the average condylar area was calculated, pre- and postoperatively. In studies that included volume measurements, the resorption rate was calculated as the difference between the percentage of formation and that of resorption of the condylar volume.

\subsection{Risk of bias in individual studies}

The studies were analyzed with the objective of identifying the risk of bias in the results and conclusions. The methodological quality of each study was assessed according to the National Council on Health and Medical Research (NHMRC) (Coleman et al., 2015) levels of evidence and notes for recommendations that established levels of evidence, according to the type of research question, considering the diagnostic accuracy, prognosis, etiology, and screening intervention. The studies were classified into levels of evidence (I, II, III-1, III-2, III-3, and IV).

\subsection{Method summary}

The comparative analysis between the condylar resorption rates was performed using the percentage difference between the averages calculated on linear or volumetric values and relapse.

\section{Results}

The electronic searches of the three databases identified 1,371 articles. Of these, 56 articles were selected after evaluation of the title and abstract, according to the inclusion and exclusion criteria, and the elimination of duplicate articles. Of these 56 articles, 50 were excluded because they did not describe the condylar resorption rate, the method of analysis used, or the validation of a new technique for 3D analysis. Thus, six articles (Kobayashi et al., 2012; Park et al., 2012; Scolozzi et al., 2013; An et al., 2014; Ueki et al., 2015; Xi et al., 2015) were selected for the qualitative analysis, and none presented sufficient data for a quantitative analysis (Fig. 1).

\subsection{Experimental design}

Of the six studies selected, three were prospective (Kobayashi et al., 2012; Scolozzi et al., 2013; Xi et al., 2015), and three were retrospective (Park et al., 2012; An et al., 2014; Ueki et al., 2015). They were published between 2012 and 2015, and the number of patients in each study ranged from 6 to 76 . They included patients with class III skeletal patterns (Park et al., 2012; An et al., 2014; Ueki et al., 2015), class II patterns ${ }^{15}$,(Scolozzi et al., 2013; Xi et al., 2015) (Table 1).

\subsection{Selection of patients}

A total of 404 mandibular condyles were evaluated in 202 patients, with a mean age of 23.3 years. The patients underwent orthognathic surgery, with a postoperative follow-up period of 12-16 months (Tables 2 and 3).

\subsection{Treatment performed}

As shown in Table 2, the treatment modalities used involved an SSRO, with or without a Le Fort I osteotomy, with fixation by bicortical screws or monocortical plates and screws. During the postoperative period, a MMF was used for 7-14 days.

Two studies (Kobayashi et al., 2012; Ueki et al., 2015) did not state which osteotomy was used in each patient (Table 2). 


\section{Condylar resorption rate}

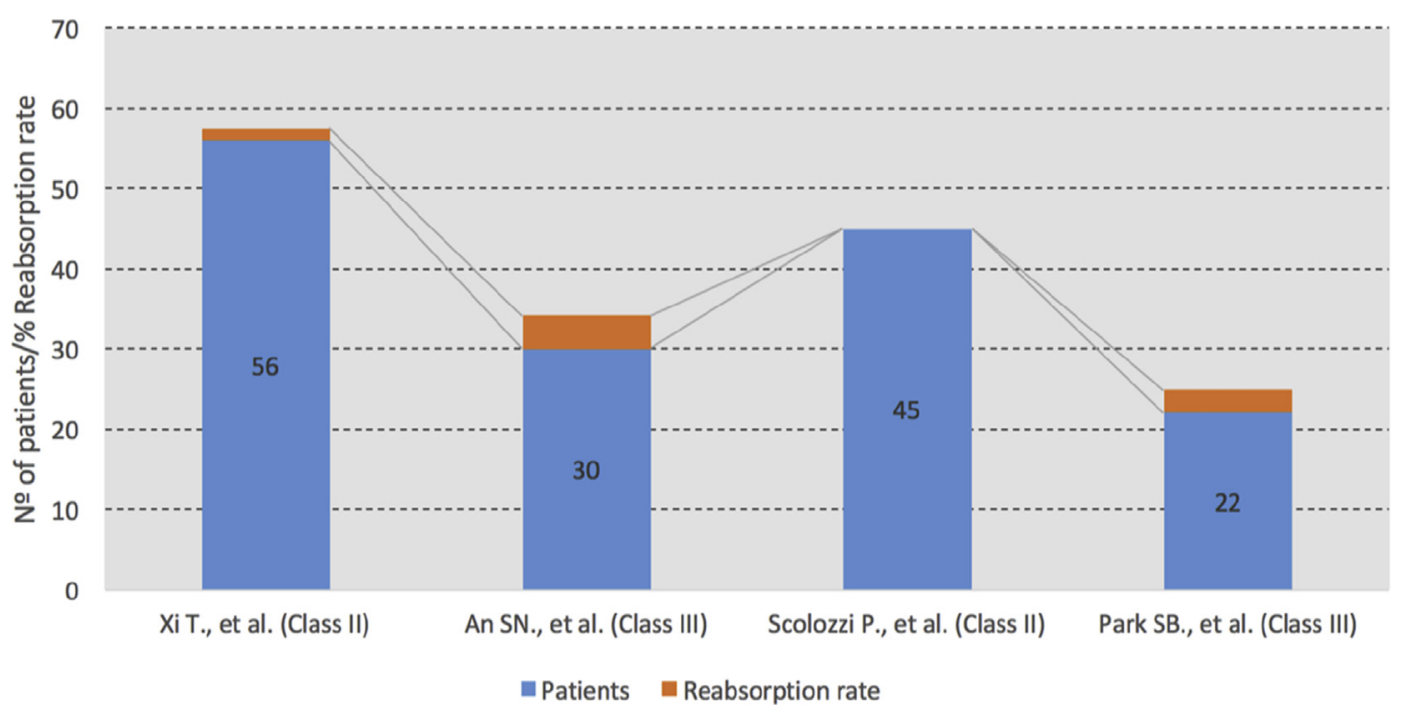

Fig. 1. PRISMA fluxogram statement of the manuscripts selected for this review.

Table 1

Characteristics of the studies.

\begin{tabular}{|c|c|c|c|c|c|c|}
\hline Authors & Year of publication & Type of study & Level of evidence & Time of study & No of patients & Type of deformities \\
\hline Ueki K., et al. & 2015 & Retrospective & III-3 & $2000-2013$ & 43 & Class III \\
\hline Xi T, et al. & 2015 & Prospective & III-3 & $2007-2011$ & 56 & Class II \\
\hline An SN., et al. & 2014 & Retrospective & III-3 & $2010-2012$ & 30 & Class III \\
\hline Scolozzi P., et al. & 2013 & Prospective & III-3 & $2007-2010$ & 45 & Class II \\
\hline Park SB., et al. & 2012 & Retrospective & III-3 & 2008-2009 & 22 & Class III \\
\hline Kobayashi T., et al. & 2012 & Prospective & III-3 & 1998-2006 & 6 & Class II \\
\hline
\end{tabular}

Table 2

Types of treatments performed.

\begin{tabular}{|c|c|c|c|c|}
\hline Authors & Osteotomy designer & Type of osteotomy fixation & Time of MMF & Follow-up \\
\hline Ueki K., et al. & SSOR/LeFort I + SSOR & Plates with monocortical screws & a & Pre-op and after 12 months \\
\hline Xi T, et al. & SSOR & Plates with monocortical screws & 7 days & Pre-op and after 12 months \\
\hline An SN., et al. & SSOR/LeFort I + SSOR & Plates with monocortical screws & & Pre-op and after 12 months \\
\hline Scolozzi P., et al. & SSOR/LeFort I + SSOR & Bicortical screws & 0 & Pre-op and after 12 months \\
\hline Park SB., et al. & LeFort I + SSRO & Bicortical screws & b & Pre-op and after 16 months \\
\hline Kobayashi T. et al. & SSOR/LeFort I + SSOR & Plates with monocortical screws or Bicortical screws & $7-14$ days & Pre-op and after 12 months \\
\hline
\end{tabular}

a Data not reported.

b There was MMF but the period of MMF was not reported.

Table 3

Number of patients and osteotomies performed.

\begin{tabular}{llllll}
\hline Authors & Male & Female & Average age & SSOR & SSOR + Le Fort I \\
\hline Ueki K., et al. & a & a & 28.3 & a & a \\
Xi T, et al. & 17 & 39 & a & 56 & 0 \\
An SN., et al. & 20 & 10 & 22,3 & 15 & 15 \\
Scolozzi P., et al. & 22 & 23 & 29,8 & 28 & 17 \\
Park SB., et al. & 11 & 11 & 20,3 & 0 & 22 \\
Kobayashi T., et al. & a & a & 21 & a & a \\
\hline
\end{tabular}

${ }^{a}$ Data not reported.

\subsection{Condylar resorption rate}

For analysis of the condylar resorption rate, five studies (Kobayashi et al., 2012; Park et al., 2012; An et al., 2014; Ueki et al., 2015; Xi et al., 2015) used computed tomography, and one (Scolozzi et al., 2013) used panoramic radiography. The analyses were
Table 4

Types of analyzes.

\begin{tabular}{llll}
\hline Authors & Image of exam & Software & Image analysis \\
\hline Ueki K., et al. & CT & Zed View versão 7.0 & Measurement \\
Xi T, et al. & CBCT & Maxilim & Superposition \\
An SN., et al. & CBCT & Rapidform XOS3 & Superposition \\
Scolozzi P., et al. & Panoramic & OxiriX & Measurement \\
Park SB., et al. & CBTC & OnDemand3D & Measurement \\
Kobayashi T., et al. & CT & a & Superposition \\
\hline
\end{tabular}

$\mathrm{CT}=$ Computed tomography.

$\mathrm{CBCT}=$ Cone Bean Computed tomography.

${ }^{\text {a }}$ Data not reported.

performed with different software; however three studies used measurements (Park et al., 2012; Scolozzi et al., 2013; Ueki et al., 2015), and three used overlays (Kobayashi et al., 2012; An et al., 2014; Xi et al., 2015) of the images. Rates of condylar resorption ranged from $0.0 \%$ to $4.2 \%$ (Tables 4 and 5 ). 
Table 5

Results of analyzes.

\begin{tabular}{lll}
\hline Authors & Resorption rate (\%) & Presence of relapse \\
\hline Ueki K., et al. & $0,0^{\mathrm{b}}$ & No \\
Xi T, et al. & $1,45^{\mathrm{b}}$ & Yes \\
An SN., et al. & $4,2^{\mathrm{c}}$ & $\mathrm{a}$ \\
Scolozzi P., et al. & $0,0^{\mathrm{b}}$ & No \\
Park SB., et al. & $2,82^{\mathrm{b}}$ & $\mathrm{a}$ \\
Kobayashi T., et al. & $\mathrm{a}$ & Yes \\
\hline a Data not reported. & \\
b Values measured through condylar area. & \\
${ }^{c}$ Values measured through condylar volume. &
\end{tabular}

Only three studies presented condylar resorption data (Park et al., 2012; An et al., 2014; Xi et al., 2015), while studies involving similar patients did not indicate the rate of condylar resorption (Scolozzi et al., 2013; Ueki et al., 2015) (Fig. 2).

\subsection{Postoperative relapse}

Four studies (Kobayashi et al., 2012; Scolozzi et al., 2013; Ueki et al., 2015; Xi et al., 2015) reported the presence or absence of postoperative relapse, however only two studies (Kobayashi et al., 2012; Xi et al., 2015) measured the extent of relapse, with values between $2 \mathrm{~mm}$ and $6.4 \mathrm{~mm}$.

\section{Discussion}

The morphological alterations of the mandibular condyles after orthognathic surgeries are extensively discussed in the literature, mainly because these alterations are likely to promote condylar resorption and, consequently, lead to relapse of skeletal dentofacial deformities (Kobayashi et al., 2012; Ueki et al., 2015; Xi et al., 2015). Generally, the studies selected for this systematic review showed that regardless of the deformity (class II or III) or the respective movement (mandibular advancement or setback, with or without movement of the maxilla), the condylar resorption rates were similar, small percentages.

For the reliability of the results, the NHMRC scale, which categorizes studies according to the type of study, allocation of patients, and the presence of control groups for comparison of the results, was used (Coleman et al., 2015. Of the six selected studies, three were prospective (Kobayashi et al., 2012; Scolozzi et al., 2013; Xi et al., 2015) (2, III-3; 1, III-2), and three retrospective (Park et al.,
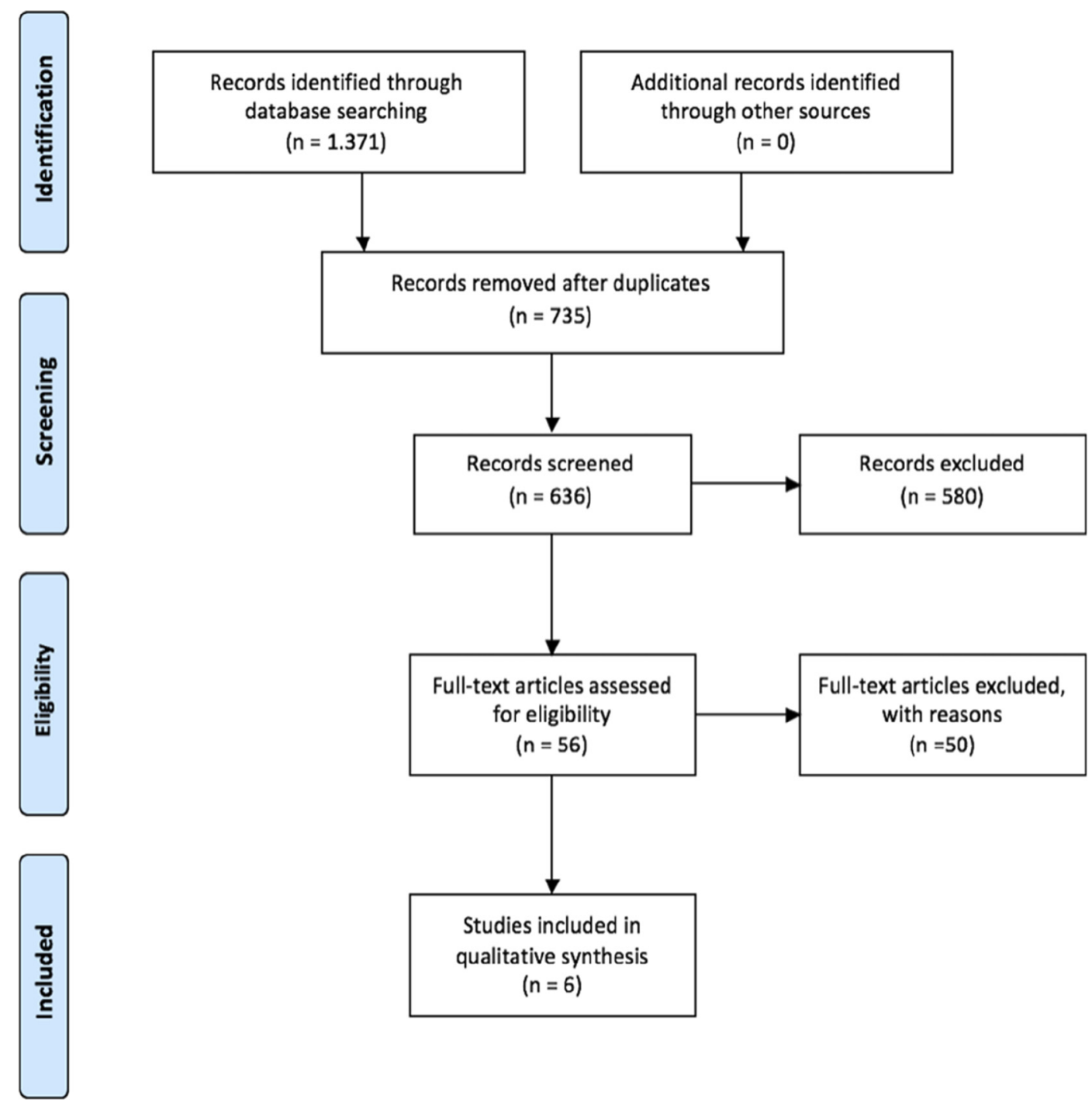

Fig. 2. Condylar resorption rate after orthognathic surgery in class II or class III patients. 
Table 6

Checklist for future study.

\begin{tabular}{ll}
\hline 1 & Gender of the patient \\
2 & Uni- or Bi-maxillary surgery \\
3 & Type of fixation \\
4 & Maxillo-mandibular fixation (use and time) \\
5 & Values of setback/advancement and clockwise/counterclockwise movements \\
6 & Follow-up more than 6 months \\
7 & Type of image exam (CT or CBCT) \\
8 & Software analysis of the images \\
9 & Metric, angular, and 3D volumetric analyses \\
10 & Symptomatology of the TMJ pre- and postoperative through of Index of Helkimo \\
11 & Presence of relapse \\
\hline
\end{tabular}

2012; An et al., 2014; Ueki et al., 2015) (3, III-3). One important point identified was that lack of RCTs limits the direct extrapolation to a clinical consensus. Therefore, future studies need to be delineated in RCT form, establishing comparison groups (class II and III, with or without asymmetry).

Even with the limitations, the analysis of the studies in this systematic review established important clinical considerations regarding the rate of condylar resorption in the postoperative period and the repercussions in the TMJ. For this type of evaluation, despite the methodological reliability scale and results, the presence of retrospective studies did not negatively influence the observations of the condylar alterations.

Important data may be lost in retrospective and prospective studies without accurate assessment. This can occur when clinical parameters are not analyzed, especially the occlusal and skeletal relapses due to the degree of condylar resorption. This reinforces the need for studies that consider the magnitude of clinical and imaging analyses.

Regarding the relapse rate, Xi et al., (2015) reported a resorption rate of $1.45 \%$. In these patients, using the horizontal measurement of the mandibular advancement in relation to the pogonion, they described relapse at a mean greater than $2 \mathrm{~mm}$. One of the factors that may have contributed to this relapse is an average value of mandibular advancement of $4.59 \mathrm{~mm}(+/-3.43 \mathrm{~mm})$. Kobayashi et al., (2012), found progressive condylar resorption in six out of 34 patients who had a mean of $12.1 \mathrm{~mm}(+/-3.9 \mathrm{~mm})$ of mandibular advancement. Ueki et al., (2015) demonstrated an average mandibular setback of $7.0 \mathrm{~mm}(+/-3.1 \mathrm{~mm})$; however, they did not report on condylar resorption or relapse.

One possible explanation for relapse in cases of pronounced mandibular advancement is the stretching of the surrounding muscle fibers. Stretching would cause pressure on the condyles, retracting them against the joint fossa, and create reabsorption during the adaptive process of the TMJ (Arnett et al., 1996a; Hwang et al., 2000). There is little evidence concerning the relation of mandibular setback to condylar resorption and relapse. Both Ueki et al. (2015) and Scolozzi et al., (2013) presented groups of skeletal class III and II patients, respectively, who underwent mandibular setbacks or advances; however, they did not describe postoperative relapses or condylar resorption rates. Therefore, the logical clinical course would be to divide the maxillary movements to allow for compensation between the mandibular setback or advancement and the three-dimensional movement of the maxilla.

The literature describes women with mandibular retrognathia, the presence of pre-treatment condylar atrophy, and subsequent condylar displacement as risk factors for the development of pathological condylar resorption (Gill et al., 2008). This assertion corroborates the findings of the studies selected in this review. Kobayashi et al., (2012) diagnosed six patients with progressive condylar resorption, of which five were women, from 34 patients who underwent mandibular advancement, and Xi et al., (2015) identified significant reductions in condylar volume $(\mathrm{p}<0.05)$ in women compared to those in men. Even though there is still no pathophysiological evidence of the relationship between the female gender and the major morphological changes of the condyles, there is strong speculation that the female hormone estrogen exerts a regulatory effect on the bone metabolism of the TMJ (Hajati et al., 2009; Gunson et al., 2012).

However, new studies investigating the pattern of altered bone metabolism using more specific imaging tests, such as bone scintigraphy and PET scanning, and evaluating levels of sex hormones and inflammatory cytokines, may contribute to the clarification of this metabolic phenomenon.

It is believed that inadequate positioning of the proximal segment during the fixation after SSRO leads to condylar remodeling in different forms and periods (Arnett, 1993). Park et al., 2012) hypothesized that there would be no significant changes between the different periods of analysis of the condyles after orthognathic surgery for correction of class III deformities; however, the hypothesis was rejected because there was a reduction of the size and a decrease of the condylar height in the postoperative period of maxillary surgeries. The regions of bone resorption and formation suggest an adaptive process of the condyles. The same authors reported that the internal rotation of the condylar head can occur both in unilateral or bilateral maxillary surgery, generating resorption in the adaptive period of the TMJ after surgery.

The studies analyzed by this review demonstrated that the type of fixation (plates or bi-cortical screws) was not a decisive factor for high rates of condylar resorption and was not associated with postoperative relapses. Independent of the technique used by experienced orthognathic surgeons for mandibular fixation, certain factors exhibit less effect on postoperative complications.

As for the accuracy of the analyses utilized by the studies, the overlapping of images, especially tomographic images used in the analysis of the condylar remodeling, are efficient; however, they are limited in the diagnosis of the resorption etiology. Thus, more specific studies are needed to differentiate the precise factors that generate the TMJ adaptation from those that generate progressive condylar resorption through similar methodologies that shows sufficient data for futures comparisons and it will be possible to perform a meta-analysis. Therefore, a checklist with mean guidelines for future clinical studies has been proposed in this paper as can be seen in Table 6.

\section{Conclusion}

According to the results obtained by this systematic review, the potential for progressive condylar resorption and relapse represent a small percentage of the patients studied. The condylar resorption rate seems to occur more in patients with a class II skeletal pattern.

\section{Conflicts of interest}

None. 


\section{References}

An SB, Park SB, Kim Y, Son WS: Effect of post-orthognathic surgery condylar axis changes on condylar morphology as determined by 3-dimensional surface reconstruction. Angle Orthod 84: 316-321, 2014

Arnett GW: A redefinition of bilateral sagittal osteotomy (BSO) advancement relapse. Am J Orthod Dentofacial Orthop 104: 506-515, 1993

Arnett GW, Milam SB, Gottesman L: Progressive mandibular retrusion-idiopathic condylar resorption. Part I. Am J Orthod Dentofacial Orthop 110: 8-15, 1996a

Arnett GW, Milam SB, Gottesman L: Progressive mandibular retrusion-idiopathic condylar resorption. Part II. Am J Orthod Dentofacial Orthop 110: 117-127, $1996 b$

Billiau AD, Hu Y, Verdonck A, Carels C, Wouters C: Temporomandibular joint arthritis in juvenile idiopathic arthritis: prevalence, clinical and radiological signs, and relation to dentofacial morphology. J Rheumatol 34: 1925-1933, 2007

Coleman K, Norris S, Weston A, Grimmer-Sommers K, Hillier S, Merlin T: NHMRC additional levels of evidence and grades for recommendations for developers of guidelines, http://wwwnhmrcgovau/_files_nhmrc/file/guidelines_stage_2_ consultation_levels_and_gradespdf

Gill DS, El Maaytah M, Naini FB: Risk factors for post-orthognathic condylar resorption: a review. World J Orthod 9: 21-25, 2008

Gunson MJ, Arnett GW, Milam SB: Pathophysiology and pharmacologic control of osseous mandibular condylar resorption. J Oral Maxillofac Surg 70: 1918-1934, 2012

Hajati AK, Alstergren P, Nasstrom K, Bratt J, Kopp S: Endogenous glutamate in association with inflammatory and hormonal factors modulates bone tissue resorption of the temporomandibular joint in patients with early rheumatoid arthritis. J Oral Maxillofac Surg 67: 1895-1903, 2009

Hoppenreijs TJ, Freihofer HP, Stoelinga PJ, Tuinzing DB, van't Hof MA: Condylar remodelling and resorption after Le Fort I and bimaxillary osteotomies in patients with anterior open bite. A clinical and radiological study. Int J Oral Maxillofac Surg 27: 81-91, 1998

Hussain AM, Packota G, Major PW, Flores-Mir C: Role of different imaging modalities in assessment of temporomandibular joint erosions and osteophytes: systematic review. Dentomaxillofac Radiol 37: 63-71, 2008

Hwang SJ, Haers PE, Sailer HF: The role of a posteriorly inclined condylar neck in condylar resorption after orthognathic surgery. J Craniomaxillofac Surg 28(2): 85-90, 2000
Katakami K, Shimoda S, Kobayashi K, Kawasaki K: Histological investigation of osseous changes of mandibular condyles with backscattered electron images. Dentomaxillofac Radiol 37: 330-339, 2008

Kobayashi T, Izumi N, Kojima T, Sakagami N, Saito I, Saito C: Progressive condylar resorption after mandibular advancement. $\mathrm{Br} J$ Oral Maxillofac Surg 50: 176-180, 2012

Moher D, Liberati A, Tetzlaff J, Altman DG, Group P: Preferred reporting items for systematic reviews and meta-analyses: the PRISMA statement. J Clin Epidemiol 62: 1006-1012, 2009

Obwegeser HL: Principles in treatment planning of facial skeletal anomalies. Clin Plast Surg 34: 585-587, 2007

Park SB, Yang YM, Kim YI, Cho BH, Jung YH, Hwang DS: Effect of bimaxillary surgery on adaptive condylar head remodeling: metric analysis and image interpretation using cone-beam computed tomography volume superimposition. J Oral Maxillofac Surg 70: 1951-1959, 2012

Pires WR, Bonardi JP, Faverani LP, Momesso GA, Munoz XM, Silva AF, et al: Late mandibular fracture occurring in the postoperative period after third molar removal: systematic review and analysis of 124 cases. Int J Oral Maxillofac Surg 46(1): 46-53, 2016

Scolozzi P, Momjian A, Courvoisier DS, Kiliaridis S: Evaluation of condylar morphology following orthognathic surgery on digital panoramic radiographs. Could methodology influence the range of "normality" in condylar changes? Dentomaxillofac Radiol 42: 20120463, 2013

Silva LD, de Lima VN, Faverani LP, de Mendonça MR, Okamoto R, Pellizzer EP: Maxillary sinus lift surgery-with or without graft material? A systematic review. Int J Oral Maxillofac Surg 45(12): 1570-1576, 2016

Tsiklakis K, Syriopoulos K, Stamatakis HC: Radiographic examination of the temporomandibular joint using cone beam computed tomography. Dentomaxillofac Radiol 33: 196-201, 2004

Ueki K, Yoshizawa K, Moroi A, Iguchi R, Kosaka A, Ikawa H, et al: Changes in computed tomography values of mandibular condyle and temporomandibular joint disc position after sagittal split ramus osteotomy. J Craniomaxillofac Surg 43: 1208-1217, 2015

Xi T, Schreurs R, van Loon B, de Koning M, Berge S, Hoppenreijs T, et al: 3D analysis of condylar remodelling and skeletal relapse following bilateral sagittal split advancement osteotomies. J Craniomaxillofac Surg 43: 462-468, 2015

Yoshioka I, Khanal A, Tominaga K, Horie A, Furuta N, Fukuda J: Vertical ramus versus sagittal split osteotomies: comparison of stability after mandibular setback. J Oral Maxillofac Surg 66: 1138-1144, 2008 